\title{
Efficacy of a Copper-Calcium-Hydroxide Solution in Reducing Microbial Plaque on Orthodontic Clear Aligners: A Case Report
}

\author{
Aida Meto ${ }^{1}$ Bruna Colombari ${ }^{2}$ Anna Castagnoli ${ }^{3}$ \\ ${ }^{1}$ School of Doctorate in Clinical and Experimental Medicine, \\ University of Modena and Reggio Emilia, Modena, Italy \\ ${ }^{2}$ Department of Surgery, Medicine, Dentistry and Morphological \\ Sciences with interest in Transplant, Oncological and Regenerative \\ Medicine, Laboratory of Microbiology and Virology, University of \\ Modena and Reggio Emilia, Modena, Italy \\ ${ }^{3}$ School of Specialization in Microbiology and Virology, University of \\ Modena and Reggio Emilia, Modena, Italy \\ ${ }^{4}$ Medical Doctor, Clinical Microbiology Laboratory, S. Agostino- \\ Estense Hospital, Baggiovara, Modena, Italy \\ ${ }^{5}$ Enzo Ferrari Engineering Department, University of Modena and \\ Reggio Emilia, Modena, Italy
}

Mario Sarti ${ }^{4}$ Lucia Denti ${ }^{5} \quad$ Elisabetta Blasi

Eur J Dent 2019;13:478-484

\begin{abstract}
Address for correspondence Elisabetta Blasi, PhD, Department of Surgery, Medicine, Dentistry and Morphological Sciences with interest in Transplant, Oncology and Regenerative Medicine, Laboratory of Microbiology and Virology, University of Modena and Reggio Emilia, Modena, Italy (e-mail: elisabetta.blasi@unimore.it).
\end{abstract}

\begin{abstract}
Keywords

- clear aligners

- copper-calciumhydroxide

- orthodontic therapy

- microbial plaque

The aim of this study was to investigate the ability of a copper-calcium-hydroxide-based compound to remove microbial plaque naturally produced onto orthodontic clear aligners. A commercially available dental paste, named Cupral, based on copper-calcium-hydroxide, was used. A healthy volunteer (female, 32 years old), undergoing orthodontic treatment with thermoplastic clear aligners was enrolled. By conventional/confocal microscopy and colony-forming unit (CFU) assay, 2-week used aligners were examined for microbial plaque, prior and following exposure to Cupral. Confocal microscopy revealed abundant plaque irregularly distributed onto the aligner surface. Following Cupral treatment, a drastic decrease occurred in plaque thickness and matrix presence. As assessed by the CFU assay, total microbial load approached $10^{9} \mathrm{CFUs/aligner,} \mathrm{with} \mathrm{slight} \mathrm{differences} \mathrm{in} \mathrm{aerobiosis} \mathrm{and} \mathrm{anaerobiosis} \mathrm{culture} \mathrm{condi-}$ tions; six macroscopically different types of colonies were detected and identified by matrix-assisted laser desorption ionization-time of flight mass spectrometry. Following Cupral treatment, microbial load dropped to undetectable levels, irrespectively of the conditions considered. Exposure of clear aligners to Cupral results in the elimination of contaminating microorganisms; the antimicrobial activity is retained up to $1.25 \%$ concentration. Overall, our data describe a novel use of Cupral, a copper-calcium-hydroxide-based compound, in daily hygiene practices with promising results.
\end{abstract}

\section{Introduction}

Notoriously, the conventional orthodontic treatment with stainless appliances ${ }^{1}$ is related to reduce patient's ability to maintain oral hygiene increasing the risk of periodontal disease via accumulation of microbial plaque..$^{2-4}$ Plaque-embedded microorganisms activate several genes involved in virulence factors ${ }^{5,6}$ reducing susceptibility to detergents or antibiotics as well as to host-mediated immune defenses. ${ }^{7-10}$

Recently, clear aligners use in dentistry allows the gradual move of teeth into an ideal position (computerized technology), minimizing microbial risk, ${ }^{11,12}$ dental trauma, and apical resorptions. ${ }^{4}$ The orthodontic protocol includes $20 / 24$ hours aligners use, removal during meals or sugar containing drinking beverages, followed by tooth and device brushing 
before re-wearing. ${ }^{11,12} \mathrm{~A}$ helpful method to visualize microbial and device-associated plaque is through the use of disclosing agents, which stain microbial pellicle. ${ }^{13,14}$

Cupral (a copper-calcium-hydroxide based compound) is a dental paste clinically used in periodontal infections, being endowed with antimicrobial activity. ${ }^{15,16}$ Moreover, recent clinical experience demonstrates that Cupral efficiently counteracts endodontic infections. ${ }^{17,18}$ Yet, its mechanisms of action have not been investigated; neither data exist on Cupral use in other dentistry fields.

The purpose of this pilot study was to investigate the ability of Cupral to remove microbial plaque, naturally produced onto orthodontic aligners. Briefly, clear aligners, regularly used by a healthy volunteer undergoing standard orthodontic therapy, were exposed or not to Cupral and assessed for total and residual microbial population, by quali-quantitative analysis.

\section{Case Report}

A healthy volunteer (female, 32-year old) undergoing orthodontic treatment for 12 months with thermoplastic clear aligners was enrolled. She started treatment on December 2017 using the Nuvola (Rome, Italy) system, that included 8 months of active treatment (replacing the aligners every 2 weeks) and 4 months of stabilization period (replacing the aligners every 2 months), the latter known as limited retention before the fixed retention applied from the clinical orthodontist. The volunteer was informed about the nature of the study, performed on individual basis, that did not directly involve her as a patient, but only the used aligners at the time of their disposal. She was asked to provide the upper and lower aligners pieces, on winter and summer months of 2018. In particular, at the times when the aligners had to be replaced with new pairs, the used ones were carefully removed from the mouth using sterile gloves and inserted in a bottle with sterile physiological saline solution $(200 \mathrm{~mL})$; then, the bottle was delivered to the Microbiology Laboratory for microbiological analysis. Totally, six couples (upper and lower) of 2-weeks-used aligners were provided, focusing on winter and summer months (January-March and June-August).

\section{Cupral}

We used a commercially available compound (HUMANCHEMIE GmbH; Alfeld, Germany), containing highly dispersed calcium and copper hydroxide. A starting solution of Cupral was prepared by diluting $1 \mathrm{~g}$ of compound in $4 \mathrm{~mL}$ of distilled water (weight/volume $25 \%$ ) sterilized by autoclave, and stored at $4^{\circ} \mathrm{C}$ prior to be used in the study. Operationally, Cupral's solution was diluted in culture medium or microbial cultures up to the final working conditions (1.25\%).

\section{Confocal Microscopy on Cupral-Treated and Untreated Aligners}

The aligners were washed with phosphate-buffered saline (PBS) at room temperature and then treated for 30 to $60 \mathrm{sec}-$ onds with plaque disclosing tablets (MGS GAP Research Co,
Ltd, Northfleet, Kent, United Kingdom) containing erythrosine red, to stain microbial plaque. Then, the aligners were further washed and brushed using running water, to mimic the daily hygiene practice. After this step, the upper and lower aligner were split in two equal pieces (right and left), transferred in two different bottles and processed in parallel; the right half upper and lower aligners were maintained in saline buffer (control group), while the left half upper and lower aligners were treated with Cupral 1.25\% (treated group), the procedure was done for 1 hour at room temperature. Finally, all the pieces were brushed again, processed using standard procedures and analyzed by transmitted illumination confocal microscopy (Nikon LV 150 Confovis Microscope, Japan).

\section{Microbiological Analysis on Clear Aligners Exposed or Not to Cupral}

The procedure is detailed in - Fig. 1 . The clear aligners were split in two equal parts and inserted in two sterile bottles, one containing saline buffer and the other Cupral $1.25 \%$. After an hour at room temperature, both bottles were sonicated at $3500 \mathrm{HZ}$ for 15 minutes to disaggregate microbial plaque. Then, the suspension was seeded uniformly onto two series of tryptic soy agar (TSA; Oxoid,Milan, Italy) and Sabouraud (SAB) plates ( $100 \mu \mathrm{L} /$ plate in triplicates), to assess total bacterial and fungal load, by colony-forming unit (CFU) assay. Two sets of TSA and SAB plates were prepared to assess microbial growth in aerobiosis and anaerobiosis conditions. Following 24 hours of incubation at $37^{\circ} \mathrm{C}$, the number of colonies/plate were counted and the results were expressed as mean of CFUx107/aligner.

According to macroscopic aspect, namely color, morphology and size, the colonies were clustered and counted. Then, to identify the isolates, representative colonies from each typology were subcultured in Columbia agar plates (OXOID; Milan, Italy); the growing colonies were then Gram stained, observed by optical microscope and identified by matrix-assisted laser desorption ionization-time of flight mass spectrometry (MALDI-TOF MS, Biomerieux, France).

\section{Optical Microscopy of the Isolated Colonies}

Representative colonies, isolated after 24 hours incubation in TSA or SAB, were observed and photographed by inverted light microscope (Nikon Eclipse TS 100, Japan).

\section{Results}

\section{Cupral Effects on Microbial Plaque Produced onto Clear Aligners: Confocal Analysis}

To investigate the structure of biofilm produced on the aligners, we performed a topographic analysis (three-dimensional [3D] mapping) by transmitted-illumination confocal microscopy, before and after Cupral treatment. - Figure 2 (upper panel) shows the overall architecture of biofilm on untreated aligner; microbial plaque and polysaccharide matrix were easily detectable (A). To evaluate biofilm thickness, a 3D reconstruction of biofilm structure was obtained from the same capture, by means of a mathematical linearization of the sample (B); according to the chromatic scale 

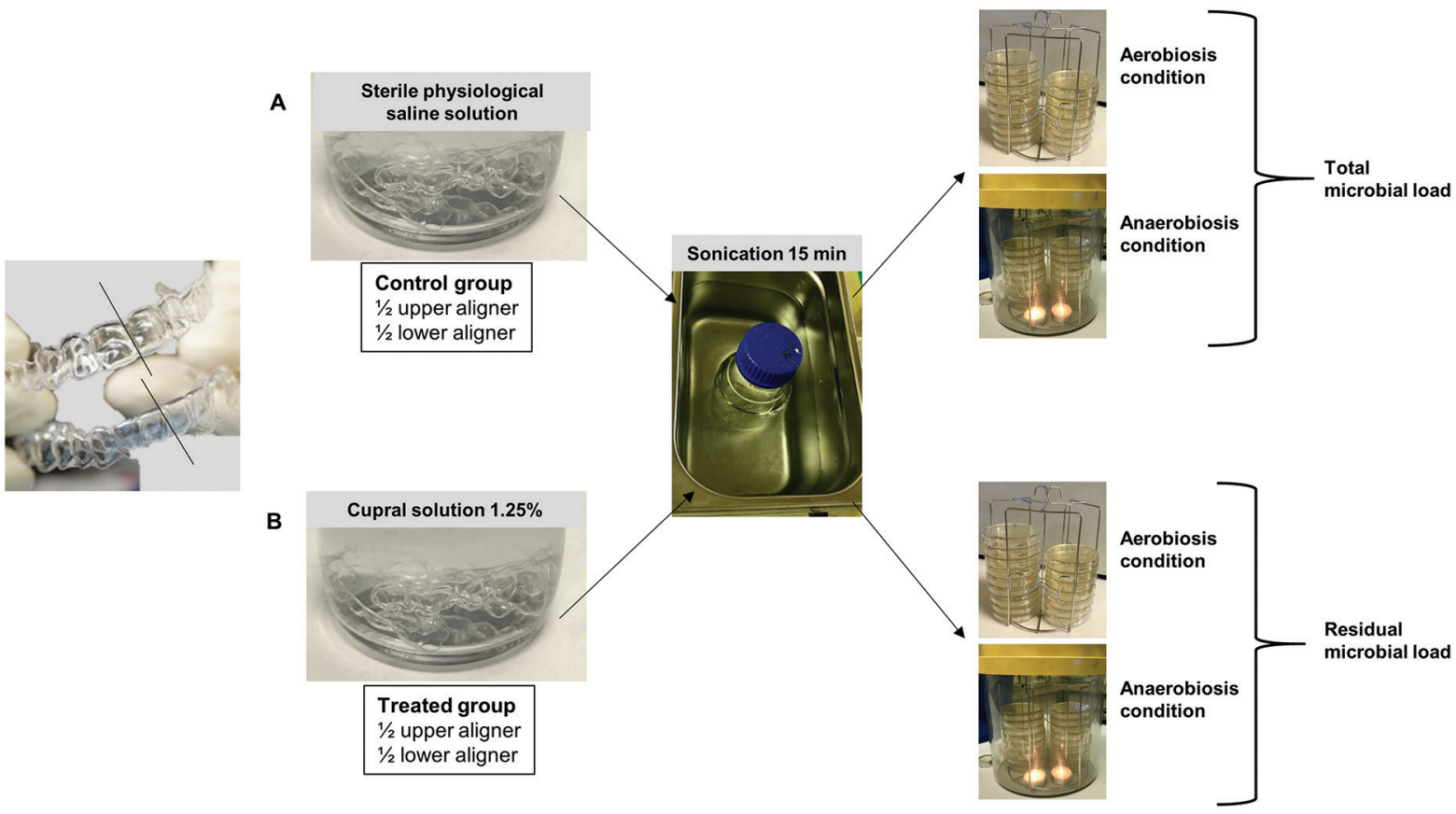

Fig. 1 Flowchart of the microbiological analysis performed on clear aligners. Two-weeks-used clear aligners (upper and lower) were split in two equal parts. A part was maintained in saline buffer (A), while the other was exposed to Cupral 1.25\% (B); both groups were incubated for 1 hour and sonicated for 15 minutes. The supernatants were harvested, diluted, and plated on TSA and SAB plates; each set of plates was incubated, under aerobiosis or anaerobiosis conditions, for 24 hours. Total and residual microbial loads were evaluated by colony-forming unit analysis; each group was tested in triplicate plates.
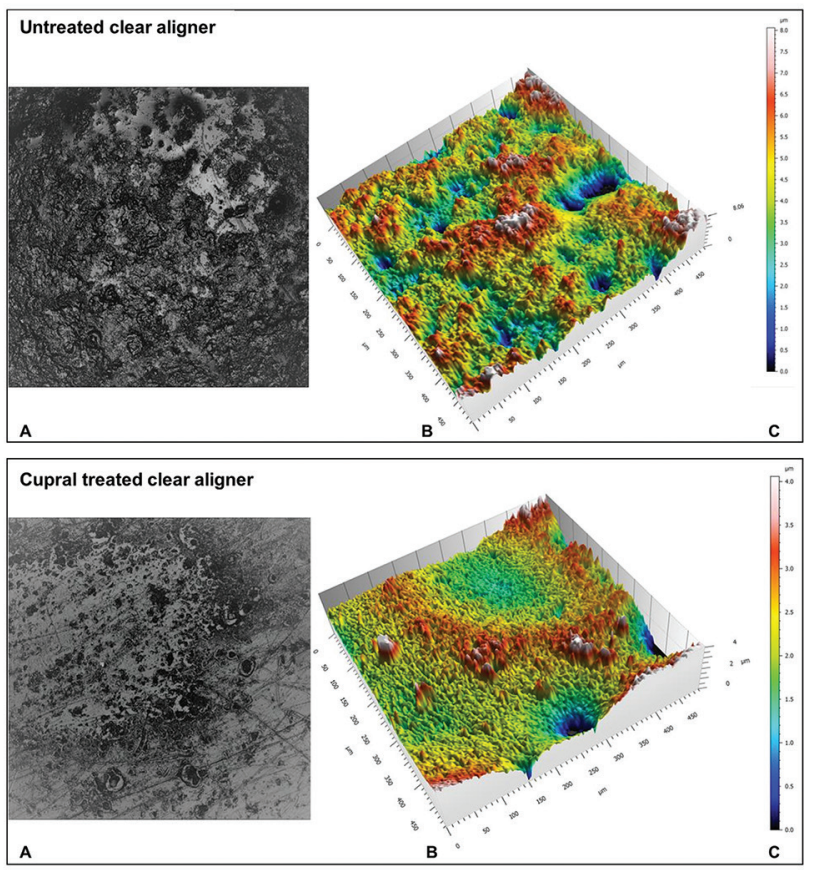

Fig. 2 Confocal microscopy of microbial plaque on orthodontic clear aligner. The upper panel shows a representative image of the untreated clear aligner; an abundant microbial biofilm is observed (A), as confirmed by the three-dimensional (3D) reconstruction of the same capture (B), according to the thickness scale (C). The lower panel shows a representative image of the Cupral-treated aligner; the device surface with minimal residual cells is observed $(\mathbf{A})$, as confirmed by the $3 \mathrm{D}$ reconstruction of the same capture (B), according to the thickness scale (C).
(C), the plaque thickness in the untreated device varied from 4.5 to $8 \mu \mathrm{m}$, as indicated by the yellow, red, and white colors. The lower panel shows an image from Cupral-treated aligner (1 hour with $1.25 \%$ ); only a scant 3D structure with minimal polysaccharide matrix was detected on the surface of the device (A). Furthermore, the 3D reconstruction (B) indicated the predominance of peaks chromatically quite uniform (mostly green and yellow colors), indicating a reduced thickness that, based on the reference scale $(\mathbf{C})$, was around 1 to $1.5 \mu \mathrm{m}$, in several areas.

\section{Cupral Effects on Clear Aligners-Associated Microbial Load: CFU Evaluation and MALDI-TOF Identification}

Microbial load was quantified in clear aligners, used for 2 weeks, during winter ( $\boldsymbol{- \text { Fig. }}$ 3) and summer months (-Fig. 4). In particular, - Figure $3 \mathrm{~A}$ indicates the total (untreated aligners) and residual (Cupral treated aligners) microbial load, using TSA and SAB medium in aerobiosis and anaerobiosis conditions. The colonies obtained in TSA ranged around 200 to $400 \mathrm{CFUx} 10^{7}$ /aligner in both conditions. In contrast, no microbial growth was detected in SAB plates in aerobiosis condition, while only few colonies were counted in anaerobiosis condition. Following Cupral treatment, no residual microbial load was ever observed, at any of the conditions tested. - Figure 3B depicts the isolated species, expressed in percent with respect to the total number of colonies. While no growth occurred in SAB under aerobiosis conditions, few colonies were detected in anaerobiosis and were identified as Rothia aeria and Streptococcus sanguinis. 


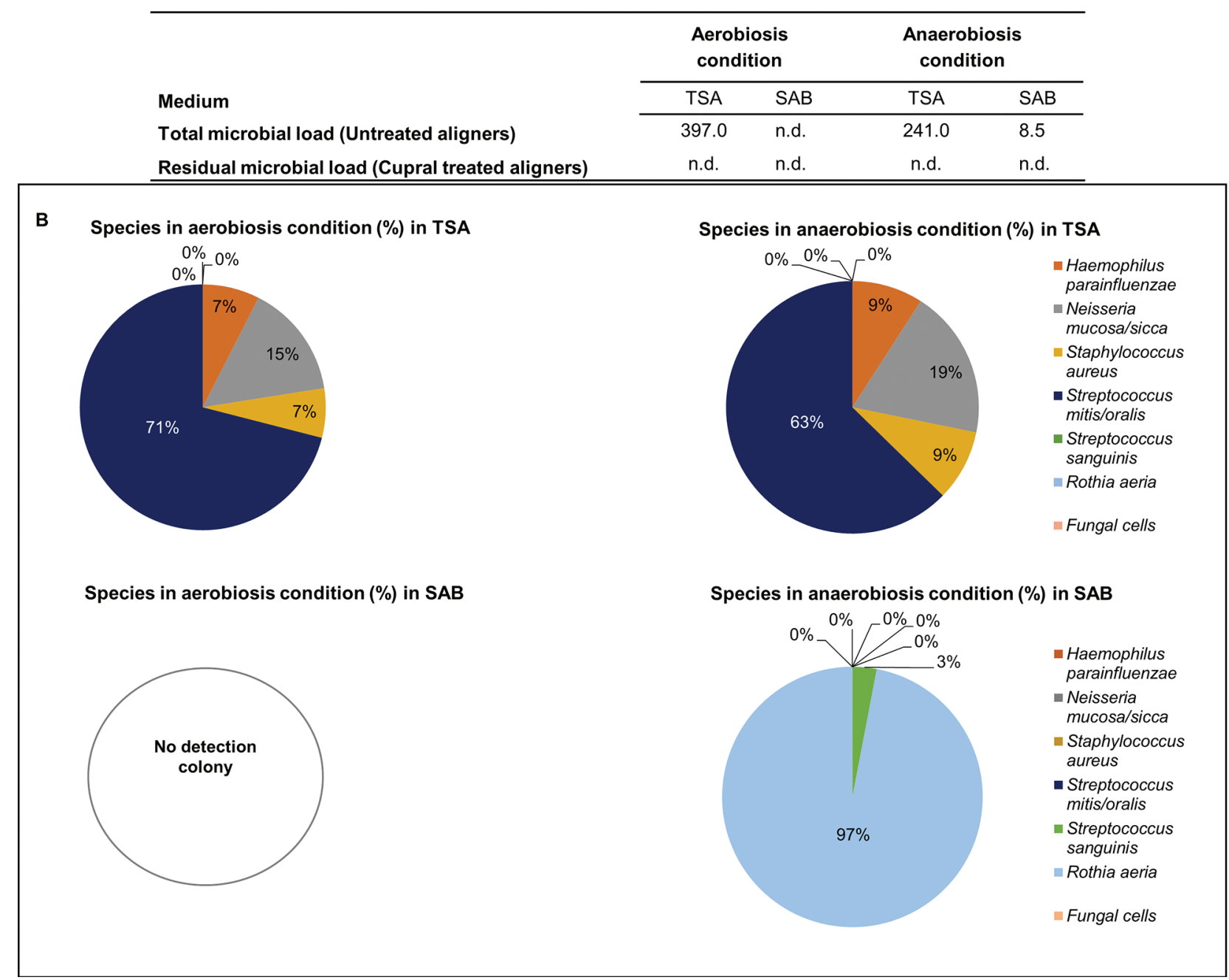

Fig. 3 TSA Total and residual microbial load detected on winter months. The clear aligners were exposed or not to Cupral 1.25\%. The colonies isolated after incubation in TSA or SAB medium were counted and clustered. The upper table shows the total and residual microbial load expressed as colony-forming units/aligners. The values represent the mean of three independent analyses, where each sample had been assessed in triplicate. The standard errors, less than 10\%, are omitted. The lower pie graphs represent the percent of species detected in aerobiosis and anaerobiosis conditions. n.d.: not detected.

As detailed in - Fig. 3B, Streptococcus mitis/oralis was the most common isolated species in TSA, both under aerobiosis and anaerobiosis conditions ( $71 \%$ and $63 \%$, respectively).

- Figure 4A shows the data obtained analyzing the aligners during summer times. The CFU/aligner in TSA ranged around 160 to $210 \mathrm{CFU} \times 10^{7}$ /aligner, while in SAB, the CFU values varied from 1.5 to $23 \mathrm{CFU} \times 10^{7} /$ aligner, in both conditions. Also, in this case, following Cupral treatment, no residual microbial load was ever observed, at any of the conditions tested. - Figure 4B displays the isolated species, expressed in percent, with respect to the total number of colonies. Here, once again Streptococcus mitis/oralis was found as the most abundant isolated species (96\% of colonies in TSA aerobiosis and $84 \%$ in TSA anaerobiosis, $99 \%$ in SAB aerobiosis and 100\% in SAB anaerobiosis).

According to morphology, color and size, the isolated species were clustered in six different types of colonies, as depicted in - Fig. 5. In particular, the colonies grown on aligners used during winter months included Gram-negative coccobacilli and diplococci, Gram-positive cocci and streptococci. On aligners used during summer months, we observed a similar trend with the most representative isolates being Gram-positive streptococci. By MALDI-TOF analysis, they were identified as $H$. parainfluenzae, N. mucosa/sicca, S. aureus, S. mitis/oralis, S. sanguinis, and R. aeria.

\section{Discussion}

Here, we show that clear aligners, regularly worn for $\sim 2$ weeks by a healthy subject, are abundantly contaminated by different bacterial species, with total microbial load ranging around $10^{9}$ per device. As predictable, microbial biofilm is naturally produced onto clear aligners; despite daily washing and brushing, the presence of microbial plaque is easily detectable by staining with plaque disclosing tablets (data not shown). According to culture-dependent analysis, Gram staining/morphology, and MALDI-TOF identification of the isolates, several bacterial species have been obtained, 
A

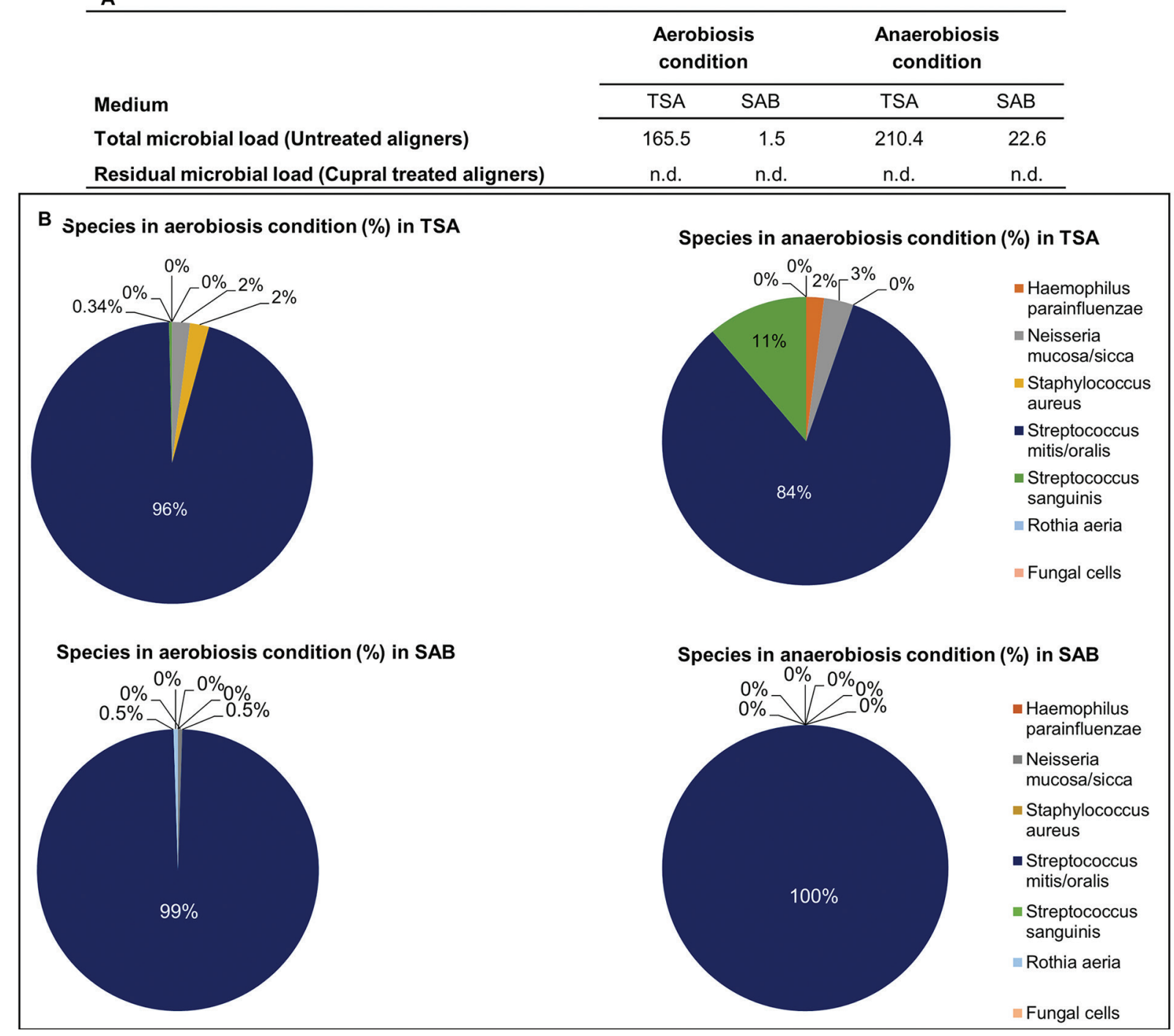

Fig. 4 Total and residual microbial load detected on summer months. The clear aligners were exposed or not to Cupral 1.25\%. The colonies isolated after incubation in SAB medium were counted and clustered. The upper table shows the total and residual microbial load expressed as colony-forming units/aligners. The values represent the mean of three independent analyses, where each sample had been assessed in triplicate. The standard errors, less than $10 \%$, are omitted. The lower pie graphs represent the percent of species detected in aerobiosis and anaerobiosis conditions. n.d.: not detected.

including Haemophilus parainfluenzae, N. mucosa/sicca, S. aureus, $S$. mitis/oralis, S. sanguinis, and $R$. aeria. In contrast, no cultivable fungal isolates have been detected, although SAB has been used as a fungi-preferential medium. Unexpectedly, under anaerobic conditions, SAB returns colonies belonging to the Streptococcus genus; likely, as facultative anaerobic species, streptococci easily grow in such conditions, because of the large amount of dextrose present in such medium, as an important element for their cellular metabolism.

It is well known that in orthodontic treatment, not only the active period but also the retention time is important because the extended periodontium must be stabilized. ${ }^{19}$ In our study, irrespective of the season time, Streptococcus mitis/ oralis happens to be the most representative species on aligners. These findings are in line with an extensive literature ${ }^{20,21}$ describing such species as commonly found in oral cavity, among the early colonizers of mucosa and dental surfaces.
In our hands, slight winter-summer variations occur. In particular, about one log reduction is observed in terms of total microbial load/aligner, when using TSA in aerobiosis conditions during summer months in comparison with winter months. Nevertheless, it is worth noting that $S$. aureus, $H$. parainfluenzae, and $N$. mucosa/sicca are vice-versa more abundant on January-March sampling $\left(10^{8} \mathrm{CFU}\right.$ and $10^{7} \mathrm{CFU} /$ aligners on winter and summer season, respectively). This result may be explained considering that such microorganisms are, indeed, easily found in the upper respiratory tract (including oral cavity) during winter season. When considering summer season, S. mitis/oralis represents the most detectable microbial species within the device-associated bacterial plaque. Overall, the relative homogeneity in terms of microbial population observed during the 1-year analysis argues on a good and regular hygiene of the oral cavity and excludes a major impact of seasonal climate on aligners colonization. 


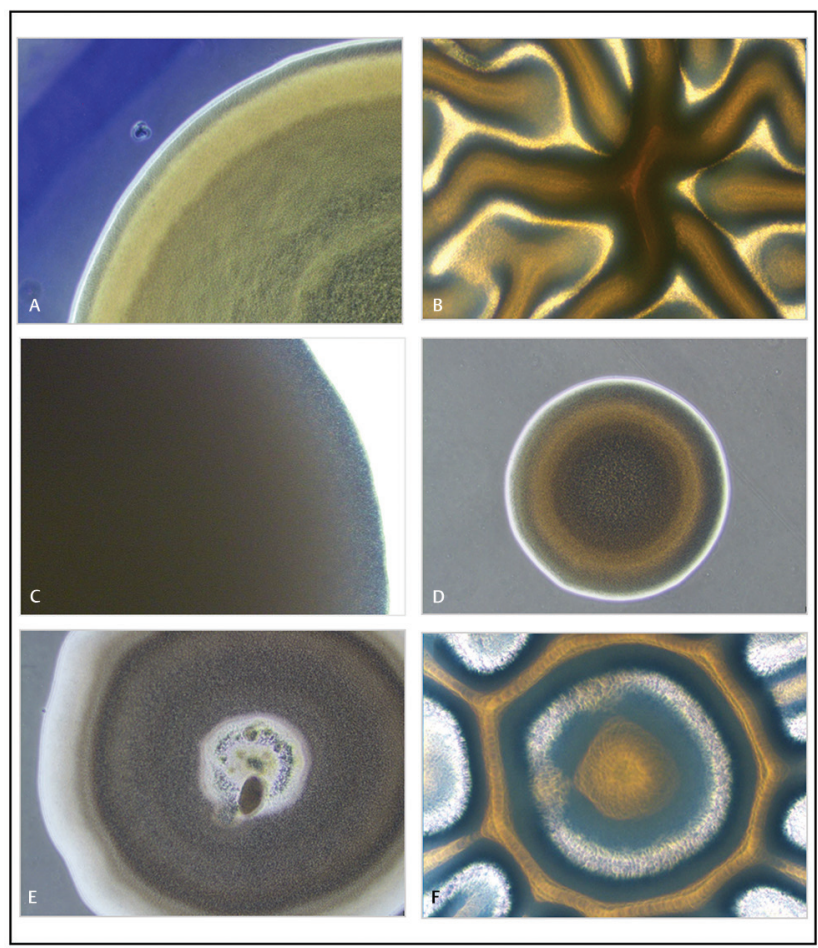

Fig. 5 Morphological peculiarities of the isolated colonies observed by inverted light microscope. Images (10x magnification) of the most representative colonies in TSA or SAB medium: (A) translucent white, coccobacilli Gram- (Haemophilus parainfluenzae); (B) wrinkled large yellow, diplococci Gram- (Neisseria mucosa/sicca); (C) large white, cocci Gram+ (Staphylococcus aureus); (D) small white, streptococci $\mathrm{Gram}^{+}$(Streptococcus mitis/oralis); (E) irregular margins, streptococci Gram+ (Streptococcus sanguinis); (F) large beige, cocci Gram+ (Rothia aeria).

Recently, we provided in vitro evidence on the efficacy of Cupral as antimicrobial compound, being effective both against planktonic and biofilm communities. ${ }^{16}$ Moreover, clinical evidence exists on the successful treatment of periodontal disease patients by Cupral. ${ }^{17,18}$ Here, we show that Cupral can be successfully employed to perform aligners hygiene; microbial contamination is totally removed, even when Cupral is diluted up to $1.25 \%$. As shown by CFU assay, no residual viable cells have ever been detectable upon Cupral treatment of the aligners. Moreover, confocal data document the abundant presence of a biofilm on aligner's surface, where microbial cells aggregate in a tridimensional architecture of irregular peaks. Interestingly, Cupral profoundly affects such biofilm; the 3D construction indicates residual material, related to debris and dead cells according to the fact that no growth has ever been obtained by the CFU assay. It is well known that not only oral hygiene but also the chemical composition of the aligners plays an important role in conditioning microbial adhesion on the devices and, in turn, persistence in the oral cavity; indeed, microcracks, abraded areas, and localized hard deposits are often visible, likely facilitating microbial establishment and long-term maintenance. ${ }^{22}$ To our knowledge, this is the first evidence describing a novel usage of Cupral, as a promising tool capable of eliminating aligner-associated microbial contamination. Such present data encourage clinical studies to establish whether the regular use of Cupral for clear aligners disinfection may allow amelioration of gingival health and integrity during orthodontic treatment.

\section{Conclusion}

Cupral, a copper-calcium-hydroxide-based compound, exerts potent effects against microbial plaque, naturally occurring on clear aligners; tridimensional biofilm structure appears profoundly affected as well as no bacterial growth ever occurs following exposure to Cupral. While expanding the knowledge on Cupral antimicrobial activity, our data open to its use as a novel tool for clear aligners daily hygiene.

\section{Conflict of Interest \\ None declared.}

\section{Acknowledgments}

The authors thank Nuvola-Rome, Italy, for providing the aligners to the volunteer.

\section{References}

1 Keim RG, Gottlieb EL, Nelson AH, Vogels DS, III. 2007 JCO orthodontic practice study. Part 1: trends. J Clin Orthod 2007;41(10):617-626

2 Gomes SC, Varela CC, da Veiga SL, Rösing CK, Oppermann RV. Periodontal conditions in subjects following orthodontic therapy. A preliminary study. Eur J Orthod 2007;29(5):477-481

3 van Gastel J, Quirynen M, Teughels W, Coucke W, Carels C. Longitudinal changes in microbiology and clinical periodontal variables after placement of fixed orthodontic appliances. J Periodontol 2008;79(11):2078-2086

4 Meto A, Blasi E, Colombari B, Tragaj E, Meto A. Endodontic treatment of apical resorptions due to fixed orthodontic treatments. Int Dent J 2017;67(1):135-198

5 Abusleme L, Dupuy AK, Dutzan N, et al. The subgingival microbiome in health and periodontitis and its relationship with community biomass and inflammation. ISME J 2013;7(5):1016-1025

6 Li L, Finnegan MB, Özkan S, et al. In vitro study of biofilm formation and effectiveness of antimicrobial treatment on various dental material surfaces. Mol Oral Microbiol 2010;25(6):384-390

7 Enaia M, Bock N, Ruf S. White-spot lesions during multibracket appliance treatment: a challenge for clinical excellence. Am J Orthod Dentofacial Orthop 2011;140(1):e17-e24

8 Richter AE, Arruda AO, Peters MC, Sohn W. Incidence of caries lesions among patients treated with comprehensive orthodontics. Am J Orthod Dentofacial Orthop 2011;139(5):657-664

9 Ahn SJ, Lim BS, Lee SJ. Prevalence of cariogenic streptococci on incisor brackets detected by polymerase chain reaction. Am J Orthod Dentofacial Orthop 2007;131(6):736-741

10 Low B, Lee W, Seneviratne CJ, Samaranayake LP, Hägg U. Ultrastructure and morphology of biofilms on thermoplastic orthodontic appliances in 'fast' and 'slow' plaque formers. Eur J Orthod 2011;33(5):577-583

11 Harradine NW. Self-ligating brackets: where are we now? J Orthod 2003;30(3):262-273

12 Lagravère MO, Flores-Mir C. The treatment effects of Invisalign orthodontic aligners: a systematic review. J Am Dent Assoc 2005;136(12):1724-1729

13 Fischman SL. Current status of indices of plaque. J Clin Periodontol 1986;13(5):371-374,379-380 
14 Marsh PD, Martin MV, Oral Microbiology. 4th ed. Oxford: Wright 1992

15 Knappwost A. Das Depotophorese-Verfahren mit Kupfer-Calciumhydroid, die zur systematischen ausheilung fuhrende alternative in der endodontie. ZWR der Zahnarzt. 1993;9:618-623

16 Meto A, Colombari B, Sala A, et al. Antimicrobial and antibiofilm efficacy of a copper/calcium hydroxide-based endodontic paste against. Staphylococcus aureus, Pseudomonas aeruginosa. and. Candida albicans. Dent Mater J 2019;38(4):591-603

17 Meto A, Meto A. Histological observation of copper sulfide. in vitro. of extracted teeth. Asian Acad Res Assoc. 2016;3(3):103-111

18 Meto A, Meto A, Tepedino M. Radiological results of apical cystic granuloma treated with Cupral. Int Dent J 2013;63(1):1-98
19 Abbate GM, Caria MP, Montanari P, et al. Periodontal health in teenagers treated with removable aligners and fixed orthodontic appliances. J Orofac Orthop 2015;76(3):240-250

20 Listgarten MA. Structure of the microbial flora associated with periodontal health and disease in man. A light and electron microscopic study. J Periodontol 1976;47(1):1-18

21 Sakamoto M, Rôças IN, Siqueira JF, Jr. Benno Y. Molecular analysis of bacteria in asymptomatic and symptomatic endodontic infections. Oral Microbiol Immunol 2006;21(2):112-122

22 Chang CS, Al-Awadi S, Ready D, Noar J. An assessment of the effectiveness of mechanical and chemical cleaning of Essix orthodontic retainer. J Orthod 2014;41(2):110-117 